ISSN 0103-5150

Fisioter. Mov., Curitiba, v. 26, n. 1, p. 87-94, jan./mar. 2013

Licenciado sob uma Licença Creative Commons

\title{
Análise comparativa da atividade elétrica do músculo multífido durante exercícios do Pilates, série de Williams e Spine Stabilization
}

\author{
Analysis of the multifidus muscle during Pilates, \\ Williams' flexion and Spine Stabilization
}

\section{Mônica Angélica Cardoso Silva ${ }^{[a]}$, Josilainne Marcelino Dias ${ }^{[b]}$, Mariana Felipe Silva ${ }^{[c]}$, Bruno Fles Mazuquin $^{[\mathrm{d}]}$, Taufik Abrão ${ }^{[\mathrm{e}]}$, Jefferson Rosa Cardoso ${ }^{[\mathrm{f}]}$}

[a] Mestranda do Programa Associado em Ciências da Reabilitação (UEL-Unopar), Londrina, PR - Brasil, e-mail: monicaa_cardoso@hotmail.com

[b] Doutoranda do Programa Associado em Educação Física (UEL-Unopar), Londrina, PR - Brasil, e-mail: lainne@hotmail.com

[c] Mestranda do Programa Associado em Ciências da Reabilitação (UEL-Unopar), Londrina, PR - Brasil, e-mail: marianaf.fisio@gmail.com

[d] Mestrando do Programa Associado em Ciências da Reabilitação (UEL-Unopar), Londrina, PR - Brasil, e-mail: bruno_mazuka@hotmail.com

[e] Pós-Doutor em Sistemas de Telecomunicações pela University of Southampton, professor associado do Departamento de Engenharia Elétrica, Universidade Estadual de Londrina (UEL) Londrina, PR - Brasil, e-mail: taufik.abrao@gmail.com

[f] Doutor em Ciências pela Universidade Federal de São Paulo (Unifesp), coordenador do Laboratório de Biomecânica e Epidemiologia Clínica, Grupo PAIFIT, Universidade Estadual de Londrina (UEL), Londrina, PR - Brasil, e-mail: jeffcar@uel.br

\section{Resumo}

Introdução: A fraqueza da musculatura paraespinhal está relacionada à etiologia da dor lombar. Atualmente existem vários métodos que apresentam exercícios para o fortalecimento dessa musculatura. Objetivos: Comparar e analisar o sinal eletromiográfico do músculo multífido bilateralmente durante exercícios do método Pilates, série de Williams e Spine Stabilization. Materiais e métodos: Participaram do estudo dez mulheres voluntárias e saudáveis que realizaram os exercícios leg pull front support modificado do Pilates, o quarto exercício da série adicional de Williams e o quadruped exercise do Spine Stabilization. 0 sinal foi normalizado pelo pico eletromiográfico da atividade dinâmica e foram ajustados para 2000 amostras por segundo e o filtro em uma frequência de passagem de 20 a $450 \mathrm{~Hz}$. A ANOVA foi utilizada para verificar diferenças entre os exercícios, o teste $t$ para amostras dependentes foi usado para comparar a ativação entre os lados direito e esquerdo do multífido para cada 
exercício. Resultados e considerações finais: Na comparação entre os exercícios, observaram-se diferenças significativas para o músculo multífido a favor do exercício do método Pilates tanto na fase concêntrica quanto na excêntrica, o que demonstra ser o exercício de melhor ativação elétrica para o músculo analisado.

Palavras-chave: Eletromiografia. Exercício. Fisioterapia.

\section{Abstract}

Introduction: The back muscle weakness is related with the etiology of low back pain. Nowadays, there are several methods the focus is on back muscle strengthening. Objectives: The purpose of this study was to compare and analyze the multifidus muscle bilaterally during exercises of the Pilates method, Spine Stabilization and Williams' flexion. Materials and methods: Ten healthy female volunteers participated in the study. They performed the leg pull front support exercise from the Pilates method, the quadruped exercise from the Spine Stabilization and the fourth exercise from the additional Williams' flexion. The signal was normalized by the electromyographic peak of the dynamic activity and was adjusted to 2000 samples/s and filter with a frequency band from 20 to $450 \mathrm{~Hz}$. ANOVA was used to verify differences between the exercises, the paired-sample t test was used to compare activation between the right and left multifidus for each exercise. Results and conclusion: When the exercises where compared, statistically significant differences were observed in the concentric and eccentric phases for the Pilates exercise, showing that this exercise is the one who activates the musculature the most between the exercises evaluated.

Keywords: Electromyography. Exercises. Physical Therapy.

\section{Introdução}

A estabilidade dinâmica da coluna vertebral está relacionada com a musculatura paraespinhal, composta pelos músculos iliocostal, longuíssimo do dorso, espinhais e multífidos $(1,2)$. Na fadiga dessa musculatura, ocorre sobrecarga sobre os elementos passivos responsáveis pela estabilidade da coluna vertebral (cápsulas, ligamentos e discos intervertebrais), o que causa danos a estruturas sensíveis à distensão e dor (3). A fadiga excessiva desses músculos paraespinhais é muitas vezes associada à dor lombar crônica (4).

Estudos biomecânicos e clínicos mostram que os músculos promovem a estabilização segmentar por meio do controle motor da região neutra e que esta pode ser recuperada dentro das limitações fisiológicas por um controle muscular efetivo $(5,6)$. Em indivíduos com lombalgia crônica observou-se, por exame de ressonância magnética, $80 \%$ de degeneração dos músculos multífidos (7). Em função desse desequilíbrio dos multífidos, diversos métodos são utilizados atualmente na prática clínica para o fortalecimento dos músculos estabilizadores da coluna, entre eles, pode-se citar o Pilates, a Série de Williams e o Spine Stabilization.
O Pilates é um método que tem sido bem difundido na atualidade e possui vantagens como estimular a circulação, melhorar o condicionamento físico, a flexibilidade, a amplitude muscular e o alinhamento postural $(8,9)$. Foi desenvolvido por Joseph Pilates durante a Primeira Guerra Mundial e reúne conceitos de ginástica, yoga, artes marciais e dança. 0 sistema básico possui exercícios para todo o corpo e foca principalmente o fortalecimento das musculaturas abdominal e paravertebral e também a flexibilidade da coluna $(9,10)$. A Série de Williams foi desenvolvida por Paul Williams, em 1937, como um programa de exercícios que tem como proposta reduzir a dor e promover estabilidade de tronco em pacientes com dor lombar crônica (11). 0 Spine Stabilization salienta a importância da força, do controle e da resistência, requerendo o uso de toda a musculatura do tronco (12). Estudos demonstram que os exercícios do método são mais eficientes em pacientes que possuem instabilidade da coluna vertebral $(13,14)$.

Na prática clínica, é necessário o conhecimento sobre quais exercícios oferecem maior dificuldade de realização para que uma progressão adequada seja realizada. Além disso, o uso de exercícios não é exclusivo à reabilitação, sendo muitas vezes utilizado na prevenção de condições dolorosas, como a lombalgia. 
Na literatura, há uma carência de estudos que comparem diferentes métodos de exercício para os extensores do tronco. Assim, o estudo tem como objetivo comparar e analisar por meio da eletromiografia de superfície o músculo multífido bilateralmente durante um exercício de Pilates, um da Série de Williams e um do Spine Stabilization em mulheres saudáveis.

\section{Método}

\section{Participantes}

A amostra foi de dez mulheres voluntárias, saudáveis, de 18 a 25 anos, com idade média de 21,5 anos (DP $=0,64$ ) e índice de massa corporal médio de 19,6 $\mathrm{kg} / \mathrm{cm}^{2}(\mathrm{DP}=0,4)$. Os critérios de exclusão foram: praticantes de atividades físicas regulares, lombalgia, protrusão de disco intervertebral, cirurgias abdominais ou de coluna prévias, espondilolistese, escoliose evidente, doenças neurológicas ou infecciosas, câncer e gravidez. As participantes assinaram o Termo de Consentimento Livre e Esclarecido. 0 estudo foi aprovado pelo Comitê de Ética em Pesquisa da Universidade Estadual de Londrina (CEP 266/07).

\section{Procedimento}

Antes do início das coletas foi realizada a limpeza com álcool 70\% e tricotomia do local. Os eletrodos foram posicionados sobre o músculo multífido no nível de L5, $3 \mathrm{~cm}$ de seu processo espinhoso seguindo a recomendação SENIAM (Surface Electromyography for the Non Invasive Assessment of Muscles). A distância intereletrodos foi de $2 \mathrm{~cm}$, seguindo a direção da fibra muscular. 0 eletrodo de referência foi posicionado no processo estiloide da ulna do membro superior não dominante. Foram realizadas três repetições de cada exercício, com intervalo de 3 segundos entre elas. Entre cada exercício foi estabelecido um intervalo de 5 minutos na tentativa de prevenir a fadiga muscular. A ordem de execução dos exercícios foi definida por sorteio simples.

Antes da execução do exercício, as participantes realizaram dois exercícios preparatórios do Pilates como aquecimento: Breathing e Prone hip extension. Os exercícios utilizados foram: Leg Pull front support (modificado), do método Pilates (Figura 1-A), o quarto exercício da série adicional, da Série de Williams (Figura 1-B) e o quadruped exercise do Spine Stabilization (Figura 1-C).

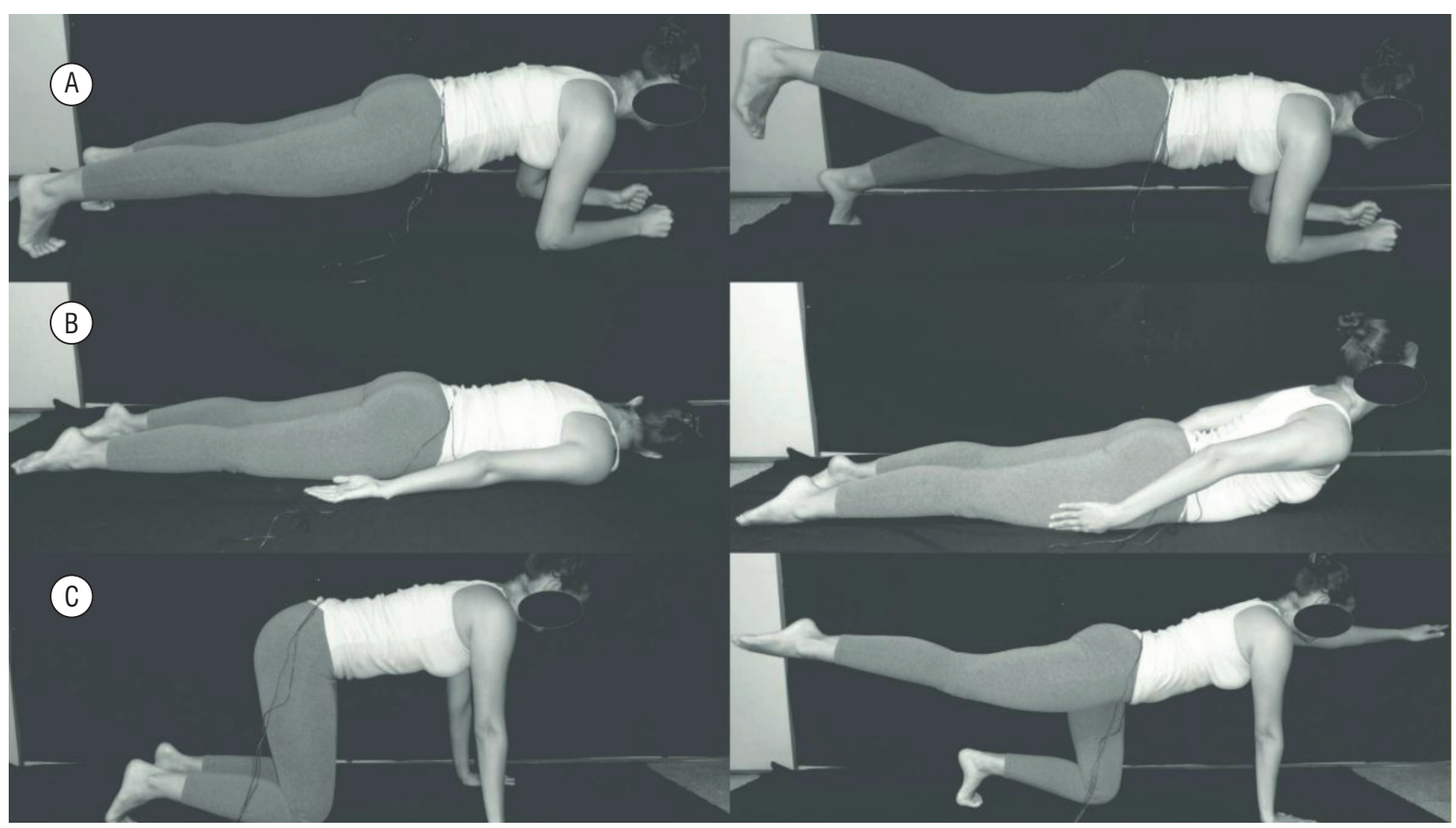

Figura 1- Exercícios analisados: Leg pull front support, do método Pilates (A); quarto exercício da série adicional, da Série de Williams (B); e Quadruped exercise, do Spine Stabilization

Fonte: Lab. PAIFIT, 2013. 
Equipamento

Foi utilizado um eletromiógrafo de superfície com 8 canais (MP150; BIOPAC System Inc., Aero Camino, CA) para obtenção dos sinais, composto por um conversor analógico-digital de 16 bits, frequência de corte com banda de 20 a $450 \mathrm{~Hz}$, ganho de amplificação total de 2000, com razão de rejeição do modo comum de $120 \mathrm{~dB}$. Eletrodos ativos bipolares foram conectados a um pré-amplificador de alta impedância $(2 \mathrm{M} \Omega)$. Todos os sinais foram coletados com frequência de amostragem de 2000 amostras por segundo por canal utilizando um programa específico para aquisição e análise dos dados (AcqKnowledge 3.9.1; BIOPAC System Inc., Aero Camino, CA). Para a diferenciação da fase concêntrica e excêntrica em cada exercício, foi utilizada uma câmera de vídeo de $30 \mathrm{~Hz}$ da marca Sony e uma placa de vídeo.

\section{Processamento do sinal EMC}

Os trechos analisados correspondem às fases concêntricas e excêntricas de cada exercício, estabelecidos por meio dos dados cinemáticos. Todos os procedimentos numéricos para obtenção dos valores de porcentagem de ativação do valor de RMS foram obtidos por meio de uma rotina matematicamente elaborada em ambiente de simulação do programa Matlab 7.6.0.324. (Mathworks, TM). Foi empregado o pico do sinal retificado da atividade dinâmica como valor de referência para a normalização do sinal.

\section{Análise Estatística}

As variáveis numéricas foram testadas quanto à distribuição de normalidade por meio do teste ShapiroWilk. A análise de variância de medidas repetidas (ANOVA) foi utilizada para verificar diferenças entre os exercícios, o teste de esfericidade de Mauchly W. foi aplicado e, quando este foi violado, correções técnicas foram realizadas utilizando o teste de GreenhouseGeisser. Quando o teste $F$ foi significante, a análise pelo teste de comparações múltiplas de Bonferroni foi aplicada. 0 teste $t$ para amostras dependentes foi usado para comparar a ativação entre os lados direito e esquerdo do multífido para cada exercício. A significância estatística foi estipulada em $5 \%$. As análises foram feitas no programa SPSS, versão 15.0

\section{Resultados}

As porcentagens de ativação variaram de 17\% a $25 \%$, com maior ativação do multífido esquerdo (ME) durante a fase concêntrica do exercício Pilates realizado com o membro inferior direito $(25,5 \%)$. Ao comparar as porcentagens de ativação entre o ME e multífido direito (MD), houve diferença com significância no exercício Spine Stabilization realizado com o lado esquerdo, tanto na fase concêntrica, com maior recrutamento do MD ( $p=0,03)$, quanto na excêntrica, que recrutou mais o ME ( $p=0,01)$; e na série de Williams, na fase excêntrica, a favor do MD $(p=0,05)$. 0 músculo ME ativou significativamente na fase excêntrica nos exercícios da série de Williams ( $\mathrm{p}=0,002)$ e Spine Stabilization $(\mathrm{p}=0,02)$ executado com o lado direito (Quadros 1 e 2). Não houve diferença significante para o exercício do Pilates tanto ao se comparar as fases quanto os músculos multífidos esquerdo e direito.

$\mathrm{Na}$ comparação entre os exercícios para o ME, foram encontradas diferenças estatisticamente significantes na fase concêntrica a favor do Pilates com o membro inferior direito $(25,5 \%)$ quando comparado aos exercícios Spine Stabilization com o membro inferior direito $(p=0,004)$, Spine Stabilization com o membro inferior esquerdo $(p=0,002)$ e Williams $(p=0,003)$. Na fase excêntrica, diferenças significantes foram encontradas a favor do Pilates com membro inferior direito $(24,6 \%)$ comparado aos exercícios Spine Stabilization com membro inferior esquerdo $(p=0,003)$ e Williams $(p=0,002)$; e entre o Pilates com membro inferior esquerdo e Williams ( $p=$ 0,005), a favor do Pilates (22,6\%) (Quadros 3 e 4).

Quadro 1 - Comparação dos valores de porcentagem de ativação normalizada (média e desvio padrão) entre os multífidos direito e esquerdo na fase concêntrica e excêntrica do Spine Stabilization e Série de Williams

(Continua)

\begin{tabular}{llc}
\hline Músculo_fase & $\overline{\mathbf{X}}(\mathrm{DP})$ & $\mathbf{p}$ \\
\hline ME_SpineD_f1 & $19,39(1,7)$ & 0,575 \\
MD_SpineD_f1 & $17,67(2,77)$ & \\
\hline ME_SpineD_f2 & $20,96(5,11)$ & 0,086 \\
MD_SpineD_f2 & $19,17(3,75)$ & \\
\hline ME_SpineE_f1 & $18,03(3,33)$ & \\
MD_SpineE_f1 & $19,70(2,57)$ & 0,032 \\
\hline
\end{tabular}


Quadro 1 - Comparação dos valores de porcentagem de ativação normalizada (média e desvio padrão) entre os multífidos direito e esquerdo na fase concêntrica e excêntrica do Spine Stabilization e Série de Williams

(Conclusão)

\begin{tabular}{lcc}
\hline Músculo_fase & $\overline{\mathbf{X}}(\mathrm{DP})$ & $\mathbf{p}$ \\
\hline ME_SpineE_f2 & $18,58(3,22)$ & 0,001 \\
MD_SpineE_f2 & $20,80(3,56)$ & \\
\hline ME_Willf1 & $18,76(1,67)$ & 0,417 \\
MD_Will_1 & $19,23(2,77)$ & \\
\hline ME_Will_2 & $17,27(2,3)$ & 0,050 \\
MD_Will_2 & $19,18(3,49)$ & \\
\hline ME_Will_f1 & $18,76(1,67)$ & 0,417 \\
MD_Will_f1 & $19,23(2,77)$ & \\
\hline ME_Will_2 & $17,27(2,3)$ & \\
MD_Will_f2 & $19,18(3,49)$ & 0,050 \\
\hline
\end{tabular}

Legenda: $\mathrm{ME}=$ Multífido esquerdo; $\mathrm{MD}=$ Multífido direito; SpineD = Spine Stabilization realizado com membro inferior direito; SpineE $=$ Spine Stabilization realizado com membro inferior esquerdo; Will = Williams; $\mathrm{f1}$ = fase concêntrica; $\mathbf{2}$ = fase excêntrica.

Fonte: Dados da pesquisa.

Nota: Diferenças significantes, quando $p<0,05$.
Quadro 2 - Comparação dos valores de porcentagem de ativação normalizada (média e desvio padrão) entre as fases concêntrica e excêntrica do Spine Stabilization realizado com membro inferior esquerdo e direito e Série de Williams

\begin{tabular}{lccc}
\hline Músculo_fase & $\begin{array}{c}\text { Fase } \\
\text { Concêntrica } \\
\bar{X}(\mathrm{DP})\end{array}$ & $\begin{array}{c}\text { Fase } \\
\text { Excêntrica } \\
\overline{\mathrm{X}}(\mathrm{DP})\end{array}$ & $\mathbf{p}$ \\
\hline ME_SpineD & $19,39(1,70)$ & $20,96(5,12)$ & 0,015 \\
MD_SpineD & $17,67(2,77)$ & $19,17(3,75)$ & 0,336 \\
ME_SpineE & $18,03(3,33)$ & $18,58(3,22)$ & 0,904 \\
MD_SpineE & $19,70(2,57)$ & $20,80(3,56)$ & 0,498 \\
ME_Will & $18,76(1,67)$ & $17,27(2,3)$ & 0,107 \\
MD_Will & $19,23(2,77)$ & $19,18(3,49)$ & 0,002 \\
\hline
\end{tabular}

Legenda: $\mathrm{ME}=$ Multífido esquerdo; $\mathrm{MD}=$ Multífido direito; SpineD = Spine Stabilization realizado com membro inferior direito; SpineE $=$ Spine Stabilization realizado com membro inferior esquerdo; Will = Série de Williams; $P$ = fase concêntrica $\times$ fase excêntrica.

Fonte: Dados da pesquisa.

Nota: Diferenças significantes, quando $p<0,05$.

Quadro 3 - Comparação dos valores de porcentagem de ativação (média e desvio padrão) normalizados entre exercícios na fase concêntrica

\begin{tabular}{|c|c|c|c|c|c|c|c|c|c|c|c|c|c|c|c|}
\hline \multicolumn{6}{|c|}{ Exercício } & \multicolumn{10}{|c|}{$P$} \\
\hline Músculo & PilatesD & PilatesE & SpineD & SpineE & Will & P1 & P2 & P3 & P4 & P5 & P6 & P7 & P8 & P9 & P10 \\
\hline ME & $\begin{array}{l}25,5 \\
(4,0)\end{array}$ & $\begin{array}{l}23,2 \\
(3,8)\end{array}$ & $\begin{array}{c}19,4 \\
(1,8)\end{array}$ & $\begin{array}{l}17,9 \\
(3,5)\end{array}$ & $\begin{array}{l}18,6 \\
(1,7)\end{array}$ & 1 & 0,049 & 0,03 & 0,03 & 0,41 & 0,16 & 0,2 & 1 & 1 & 1 \\
\hline MD & $\begin{array}{l}23,2 \\
(5,8)\end{array}$ & $\begin{array}{l}23,7 \\
(2,0)\end{array}$ & $\begin{array}{l}18,2 \\
(2,2)\end{array}$ & $\begin{array}{l}19,1 \\
(1,9)\end{array}$ & $\begin{array}{l}19,4 \\
(2,9)\end{array}$ & 1 & 0,55 & 0,74 & 0,57 & 0,01 & 0,02 & 0,01 & 1 & 1 & 1 \\
\hline
\end{tabular}

Legenda: $\mathrm{ME}=$ Multífido esquerdo; $\mathrm{MD}=$ Multífido direito; PilatesD $=$ Pilates realizado com membro inferior direito; PilatesE $=$ Pilates realizado com membro inferior esquerdo; SpineD $=$ Spine Stabilization realizado com membro inferior direito; SpineE $=$ Spine Stabilization realizado com membro inferior esquerdo; Will $=$ Williams; $\mathrm{P} 1=$ PilatesD $\times$ PilatesE; $\mathrm{P} 2=$ PilatesD $\times$ SpineD; $\mathrm{P} 3=$ PilatesD $\times$ SpineE; $\mathrm{P} 4=$ PilatesD $\times$ Will; P5 $=$ PilatesE $\times$ SpineD; P6 $=$ PilatesE $\times$ SpineE; P7 $=$ PilatesE $\times$ Will; $\mathrm{P} 8=$ SpineD $\times$ SpineE; $\mathrm{P} 9=$ SpineD $\times$ Will; $\mathrm{P} 10=$ SpineE $\times$ Will .

Fonte: Dados da pesquisa.

Nota: Diferenças significantes, quando $p<0,05$. 
Quadro 4 - Comparação dos valores de porcentagem de ativação (média e desvio padrão) normalizados entre exercícios na fase excêntrica

\begin{tabular}{|c|c|c|c|c|c|c|c|c|c|c|c|c|c|c|c|}
\hline \multicolumn{6}{|c|}{ Exercício } & \multicolumn{10}{|c|}{$p$} \\
\hline Músculo & PilatesD & PilatesE & SpineD & SpineE & Will & $P 1$ & P2 & P3 & P4 & P5 & P6 & P7 & P8 & $P 9$ & P10 \\
\hline ME & $\begin{array}{l}24,7 \\
(2,3)\end{array}$ & $\begin{array}{l}22,7 \\
(2,9)\end{array}$ & $\begin{array}{l}20,9 \\
(5,4)\end{array}$ & $\begin{array}{l}18,3 \\
(3,3)\end{array}$ & $\begin{array}{l}17,6 \\
(2,2)\end{array}$ & 0,68 & 1 & 0,3 & 0,002 & 1 & 0,28 & 0,05 & 1 & 1 & 1 \\
\hline MD & $\begin{array}{c}24,8 \\
(4,25)\end{array}$ & $\begin{array}{l}24,7 \\
(3,8)\end{array}$ & $\begin{array}{c}19,1 \\
(4,0)\end{array}$ & $\begin{array}{l}20,5 \\
(3,6)\end{array}$ & $\begin{array}{l}19,3 \\
(3,7)\end{array}$ & 1 & 0,5 & 0,28 & 0,57 & 0,39 & 0,38 & 0,11 & 1 & 1 & 1 \\
\hline
\end{tabular}

Legenda: ME = Multífido esquerdo; MD = Multífido direito; PilatesD = Pilates realizado com membro inferior direito; PilatesE = Pilates realizado com membro inferior esquerdo; SpineD = Spine Stabilization realizado com membro inferior direito; SpineE = Spine Stabilization realizado com membro inferior esquerdo; Will = Williams; P1 = PilatesD $\times$ PilatesE; P2 = PilatesD $\times$ SpineD; P3 = PilatesD $\times$ SpineE; P4 $=$ PilatesD $\times$ Will; P5 $=$ PilatesE $\times$ SpineD; P6 $=$ PilatesE $\times$ SpineE; P7 $=$ PilatesE $\times$ Will; P8 $=$ SpineD $\times$ SpineE; P9 $=$ SpineD $\times$ Will; P10 $=$ SpineE $\times$ Will.

Fonte: Dados da pesquisa.

Nota: Diferenças significantes, quando $p<0,05$.

Na comparação entre os exercícios para o MD, diferenças significantes foram encontradas a favor do Pilates com o membro inferior esquerdo $(23,7 \%)$ quando comparado com o Spine Stabilization com o membro inferior direito $(\mathrm{p}=0,001)$, Spine Stabilization com o membro inferior esquerdo $(\mathrm{p}=0,002)$ e Williams ( $p=0,003$ ) (Quadros 3 e 4).

\section{Discussão}

O exercício leg pull front support adaptado do método Pilates foi o que apresentou maior porcentagem de ativação entre os exercícios avaliados $(23,2$ a 25,5\%). Menacho et al. (15) avaliaram a ativação da musculatura extensora em três exercícios de Pilates: swimming (61\%), single leg kick (19\%) e double leg kick (47\%). Estes dados confirmam que os exercícios do Pilates podem promover boa ativação desse grupo muscular.

Para o pós-operatório de microdiscectomia, Flannagan et al. (16) e Selkovitz et al. (17) apresentaram em seus estudos um protocolo com exercícios para tronco e membros inferiores. 0 protocolo apontou várias séries de exercícios e suas progressões, entre elas, uma em que se inicia com o quadruped exercise e, em seu maior grau de dificuldade, um exercício semelhante ao leg pull front support, corroborando com nossos resultados de que o exercício baseado no método Pilates apresenta uma porcentagem de ativação maior que a Spine Stabilization.

Como músculo multífido tem a capacidade de controlar o movimento de um segmento móvel ileso da coluna lombar e restaurar o controle desse segmento após uma lesão (18), é importante investigar a ativação desse músculo aplicada à reabilitação. Conforme os resultados encontrados nesse estudo, o ideal seria aplicar o exercício do método Pilates como evolução dos exercícios do Spine Stabilization e da Série de Williams.

Em um estudo (19) americano foram encontradas porcentagens de contração isométrica voluntária máxima (CIVM) de 56\% para multífido esquerdo e 50\% para multífido direito em um exercício de extensão de tronco (Série de Williams). Entretanto, esse foi realizado mantendo o quadril equilibrado em uma bola suíça e os pés no chão, demonstrando que variações do exercício básico utilizado nesse trabalho podem promover maiores porcentagens de ativação da musculatura. Ainda nesse trabalho, o autor classifica o exercício quadruped como um exercício moderado, pois, como em nosso estudo, obteve uma porcentagem de ativação menor que $50 \%$.

Como visto anteriormente, no exercício do Spine Stabilization realizado com o membro inferior esquerdo em sua fase excêntrica, o multífido direito foi o mais 
recrutado ao comparar a porcentagem do valor de RMS, isso se deve possivelmente ao trabalho de estabilização feito pelo músculo para que o movimento com o membro inferior contralateral possa ocorrer. Em uma análise da arquitetura muscular do multífido, Ward et al. (20) concluíram que esse músculo tem uma estrutura anatômica propícia para agir como estabilizador quando grandes forças são produzidas, como por exemplo, a extensão do membro, além de que o multífido fornece uma fonte essencial de extensão e torque de rotação para a base da coluna (21).

\section{Conclusão}

Os resultados apontaram que exercício do Pilates, quando comparada a porcentagem de ativação, proporciona uma atividade muscular maior que nos demais exercícios analisados. Portanto, na prática clínica, considerando esses resultados, é sugerido que se utilize o exercício do Pilates em uma fase mais avançada de tratamento. Além disso, foi observada a importância do músculo multífido em sua ação estabilizadora.

\section{Agradecimentos}

Os autores agradecem à Fisioterapeuta Ana Paula Rossetto G. Cardoso pelas orientações quanto aos exercícios e leitura crítica do manuscrito e também agradecem ao CNPq pelo Edital MCT/CNPq 12/2010, à Fundação Araucária pelo Edital Programa de Apoio à Pesquisa Básica e Aplicada (14/2008) e pela Bolsa Produtividade em Pesquisa para os dois últimos autores.

\section{Referências}

1. Bogduk N, Macintosh JE, Pearcy MJ. A universal model of the lumbar back muscles in the upright position. Spine. 1992;17(8):897-913. doi:10.1097/ 00007632-199208000-00007.

2. Kendall F, McCreary EK, Provance P, Rodgers MM, Romani WA. Músculos provas e funções. 5. ed. São Paulo: Manole; 2007.
3. Gonçalves M, Barbosa FSS. Análise de parâmetros de força e resistência dos músculos eretores da espinha lombar durante a realização de exercício isométrico em diferentes níveis de esforço. Rev Bras Med Esporte. 2005;11(2):109-14. doi:10.1590/ S1517-86922005000200003.

4. Holmstrom E, Moritz U, Andersson M. Trunk muscle strength and back muscle endurance in construction workers with and without low back disorders. Scand J Rehabil Med. 1992;24(1):3-10. PMid:1534932.

5. Danneels LA, Vanderstraeten GG, Cambier DC, Witvrouw EE, Bourgois J, Dankaerts W, et al. Effects of three different training modalities on the cross sectional area of the lumbar multifidus muscle in patients with chronic low back pain. Br J Sports Med. 2001;35(3):186-91. doi:10.1136/bjsm.35.3.186.

6. Suni J, Rinne M, Natri A, Statistisian MP, Parkkari J, Alaranta H. Control of the lumbar neutral zone decreases low back pain and improves self-evaluated work ability: a 12-month randomized controlled study. Spine. 2006;31:E611-20. doi:10.1097/01.brs. 0000231701.76452 .05 .

7. Kader D, Wardlaw D, Smith F. Correlation between the MRI changes in the lumbar multifidus muscles and leg pain. Clin Radiol. 2000;55:145-9. doi:10.1053/ crad.1999.0340.

8. Sacco ICN, Andrade MS, Souza PS, Nisiyama M, Cantuária AL, Maeda FYI, et al. Método pilates em revista: aspectos biomecânicos de movimentos específicos para reestruturação postural - estudos de caso. R Bras Ci e Mov. 2005;13:65-78.

9. Dorado C, Calbet JAL, Lopez-Gordillo A, Alayon S, Sanchis-Moysi J. Marked effects of Pilates on the abdominal muscles: a longitudinal MRI study. Med Sci Sports Exerc. 2012;44(8):1589-94. doi:10.1249/ MSS.0b013e31824fb6ae.

10. Kolyniak IEG, Cavalcanti SMB, Aoki MS. Avaliação isocinética da musculatura envolvida na flexão e extensão do tronco: efeito do método Pilates. Rev Bras Med Esporte. 2004;10(6):487-90. doi:10.1590/ S1517-86922004000600005.

11. Blackburn SE, Portney LG. Electromyographic activity of back musculature during Williams' flexion exercises. Phys Ther. 1981;61(6):878-5. PMid:6454146. 
12. McGill SM, Karpowicz A. Exercises for spine stabilization: motion/motor patterns, stability progressions, and clinical technique. Arch Phys Med Rehabil. 2009;90(1):118-26. doi:10.1016/j.apmr.2008.06.026.

13. Souza GM, Baker LL, Powers CM. Electromyographic activity of selected trunk muscles during spine stabilization exercises. Arch Phys Med Rehabil. 2001;82(11):1551-7. doi:10.1053/apmr.2001.26082.

14. Fritz JM, Whitman JM, Childs JD. Lumbar spine segmental mobility assessment: an examination of validity for determining intervention strategies in patients with low back pain. Arch Phys Med Rehabil. 2005;86:1745-52. doi:10.1016/j.apmr.2005.03.028.

15. Menacho MO, Obara K, Conceição JS, Chitolina ML, Krantz DR, Silva AS, et al. Electromyographic effect of mat Pilates exercise on the back muscle activity of healthy adult females. J Manipulative Physiol Ther. 2010;33(9):672-8. doi:10.1016/j.jmpt.2010.08.012.

16. Flanagan SP, Kulig K, Clinresnet P. Time courses of adaptation in lumbar extensor performance of patients with a single-level microdiscectomy during a physical therapy exercise program. J Orthop Sports Phys Ther. 2010;40(6):336-44. PMid:20479532.

17. Selkowitz DM, Kulig K, Clinresnet P, Poppert EM, Flanagan SP, Matthews ND, Beneck GJ, et al. The immediate and long-term effects of exercise and patient education on physical, functional, and quality-of-life outcome measures after single-level lumbar microdiscectomy: a randomized controlled trial protocol. BMC Musculoskelet Disord, 2006; 7:70. doi:10.1186/1471-2474-7-70.
18. Macdonald DA, Moseley GL, Hodges PW. The lumbar multifidus: does the evidence support clinical beliefs? Man Ther. 2006;11(4):254-63. doi:10.1016/j. math.2006.02.004.

19. Ekstrom RA, Osborn RW, Hauer PL. Surface electromyographic analysis of the low back muscles during rehabilitation exercises. J Orthop Sports Phys Ther. 2008;28:736-45.

20. Ward SR, Kim CW, Eng CM, Gottschalk IV LJ, Tomiya A, Garfin, SR, et al. Architectural analysis and intraoperative measurements demonstrate the unique design of the multifidus muscle for lumbar spine stability. J Bone Joint Surg Am. 2009;91(1):176-85. doi:10.2106/JBJS.G.01311.

21. Tateuchi H, Taniguchi M, Mori N, Ichihashi N. Balance of hip and trunk muscle activity is associated with increased anterior pelvic tilt during prone hip extension. J Electromyogr Kinesiol. 2012;22(3):391-7. doi:10.1016/j.jelekin.2012.03.003.

Recebido: 19/07/2012

Received: 07/19/2012

Aprovado: $10 / 01 / 2013$

Approved: 01/10/2013 